\title{
Morphological stability of epitaxial thin elastic films by van der Waals force
}

\author{
Ya-Pu Zhao
}

Summary The morphological stability of epitaxial thin elastic films on a substrate by van der Waals force is discussed. It is found that only van der Waals force with negative Hamaker constant $(A<0)$ tends to stabilize the film, and the lower bound for the Hamaker constant is also obtained for the stability of thin film. The critical value of the undulation wavelength is found to be a function of both film thickness and external stress. The charateristic time-scale for surface mass diffusion scales to the fourth power to the wavelength of the perturbation.

Keywords Epitaxial Thin Film, Elasticity, Van der Waals Force, Perturbation, Morphological Stability

1

Introduction

Epitaxial thin film structures have found wide applications in many electronic, optoelectronic and microsystem devices. These films, with thickness typically ranging from a few nanometers to a few micrometers, are usually subject to very large stresses due to the lattice mismatch and thermal expansion coefficient mismatch between the thin film and substrate. The morphological stability of thin elastic films has been studied extensively in both mechanics and materials sciences (e.g., see a recent review [1]). It is well agreed that the surface roughening of thin films, as shown in Fig. 1, [2], as well as other kinds of microstructures, [3], are consequences of the competition between the strain energy and the surface energy in heterostructures, [1]. The basic length-scale of roughening corresponds to the ratio between the surface energy density and the strain energy density of the film, [4], i.e.

$l_{\mathrm{cr}} \sim \frac{E \gamma_{0}}{\sigma^{2}}$,

where $E$ is Young's modulus, $\gamma_{0}$ is the surface energy density and $\sigma$ is the stress, such as residual stress caused by lattice misfit or thermal expansion coefficient misfit between the elastic thin film and substrate. It is noted that such a property is common for the morphological evolution of microstructures such as thin films, whiskers, pore channels, voids, etc..

The types of forces that influence micro-scale devices are different from those that influence conventional-scale ones. This is because the size of a physical system bears a significant influence on the physical phenomena that dictate the dynamic behavior of that system. With the miniaturization of structures, interfacial forces, including capillary force, electrostatic and van der Waals forces, will be important, and even become dominant. Intermolecular spacing is important when van der Waals forces play a decisive role in the adhesive contact

Received 4 December 2000; accepted for publication 31 July 2001

$\mathrm{Ya}-\mathrm{Pu} \mathrm{Zhao}$

State Key Laboratory of Nonlinear Mechanics (LNM),

Institute of Mechanics, Chinese Academy of Sciences, Beijing 100080, P.R. China

e-mail: yzhao@lnm.imech.ac.cn

The supports given by the China National " 973 " project and National Natural Science Foundation are gratefully acknowledged. The author is grateful to the two reviewers of the AAM for their insight and valuable comments. 


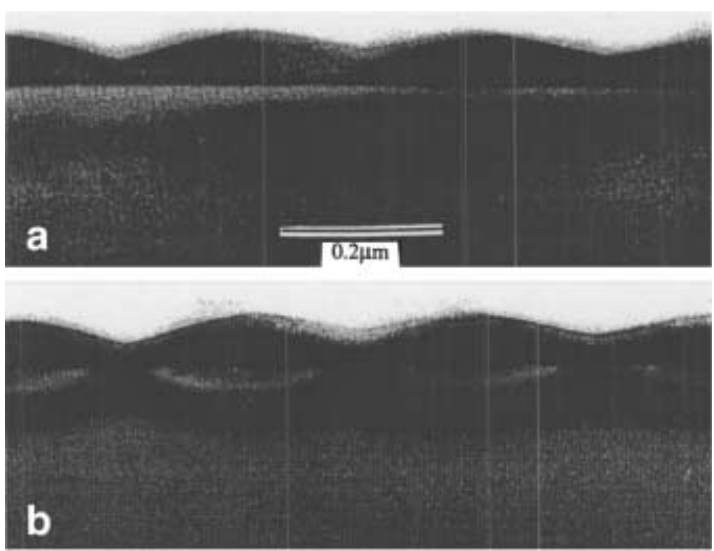

Fig. 1. Undulation observed on the surface of a silicon-germanium layer grown on (001) silicon under different operating reflections: a only undulations are visible, and $\mathbf{b}$ both undulations and strain contrast are seen

of two bodies. For example, larger-scale systems are influenced by inertia effects to a much greater extent than smaller-scale systems, while smaller systems are influenced by surface effects. As an illustration, a ball bearing $1 \mathrm{~cm}$ in diameter would experience an adhesion force to a dirty surface several orders of magnitude smaller than the force of gravity. By contrast, a bearing $1 \mu \mathrm{m}$ in diameter experiences an adhesion force more than a million times greater than the force of gravity, since the adhesion force falls linearly with size whereas the gravitional force falls with the size cubed. Given the dimensions of most micro-devices, gravity and other body forces are weaker by many orders of magnitude than the intermolecular forces.

There are four different kinds of binding types for solid, namely, ionic binding, covalent binding, metallic binding and van der Waals binding. The van der Waals dispersion force between two bodies is caused by mutual electric interaction of the induced dipoles in the two bodies, [5]. The van der Waals force consists of three parts: Keesom orientation interaction, Debye induction and dispersion forces. Dispersion forces, generally, dominate over orientation and induction forces, except for strongly polar molecules. Disperson forces play an important role in phenomena such as adhesion, surface tension, physical absorption, wetting, the properties of gases, liquids and thin films, the strength of solids, the flocculation of particles in liquids, and the structures of condensed macromolecules such as proteins and polymers. The main features of dispersion forces may be summarized as follows:

(1) They are long-range forces and, depending on the situation, can be effective from large distances (greater than $10 \mathrm{~nm}$ ) down to interatomic spacings (about $0.2 \mathrm{~nm}$ ).

(2) These forces may be repulsive or attractive, and, in general, the dispersion force between two molecules or large particles does not follow a simple power law.

(3) Dispersion forces not only bring molecules together but also tend to mutually align or orient them, though this orienting effect is usually weak.

(4) The dispersion interaction of two bodies is affected by the presence of other bodies nearby. This is known as the non-additivity of an interaction.

Dispersion forces are quantum mechanical in origin. All interaction laws underlying van der Waals force depend on geometrical features of the bodies and on the Hamaker constant $A$, that includes all physico-chemical information

$A=\pi^{2} C \rho_{1} \rho_{2}$,

in which $C$ is the constant in the atom-atom pair potential, and $\rho_{1}$ and $\rho_{2}$ are the numbers of atoms per unit volume of two bodies. Typical values of the Hamaker constant of condensed phases (most solids and liquids) lie in the range $(0.4-4) \times 10^{-19} \mathrm{~J}$ in vacuum.

The influence of long-range forces on the stability of epitaxial films has been studied in [6], the critical film thickness was obtained by considering van der Waals force interaction.

However, to the author's knowledge, no work has been found concerning the influence of van der Waals force on the characteristics of the perturbation of the film surface, which would be quite important for the stability and surface diffusion time of the film. The objective of the present paper is to study the influence of van der Waals force on the stability of epitaxial thin films on substrate. 


\section{Static global energetic conservation due to perturbation of film surface}

Consider a thin epitaxial film of nominal thickness $h_{0}$ on a flat substrate. For simplicity and without loss of generality, $[1,6,7]$, suppose the film and the substrate have the same elastic constants (the film and substrate materials are not necessarily the same). From Fig. 1 it is known that a one-dimensional disturbance changes the shape to

$h(x)=h_{0}+q \cos \left(\frac{2 \pi x}{\lambda}\right)$,

where $q$ is a small perturbation and $\lambda$ is the wavelength, as shown in Fig. 2. The $x$-axis coincides with the film-substrate interface. Such a surface profile conserves mass, since

$\frac{1}{\lambda} \int_{0}^{\lambda} h(x) \mathrm{d} x=h_{0}$

Using the cosine profile (2), the increasing of the surface length in the $x$-axis direction, $\Delta \lambda$, in one period in the surface area is given as

$\Delta \lambda=\int_{0}^{\lambda} \sqrt{1+\left(\frac{2 q \pi}{\lambda}\right)^{2} \sin ^{2}\left(\frac{2 \pi x}{\lambda}\right)} \mathrm{d} x-\lambda$.

Assuming that $q \ll \lambda$, the increasing of the surface length in the $x$-axis can be simplified to

$\Delta \lambda=2\left(\frac{q \pi}{\lambda}\right)^{2} \int_{0}^{\lambda} \sin ^{2}\left(\frac{2 \pi x}{\lambda}\right) \mathrm{d} x=\frac{q^{2} \pi^{2}}{\lambda}$.

Therefore, as the first approximation, the total surface-energy change due to the pertubation is

$\Delta W_{s}=\frac{\gamma_{0} q^{2} \pi^{2}}{\lambda}$

where $\gamma_{0}$ is the surface energy density.

The sources of defects in epitaxial thin films can be broadly split into two different categories: growth-process-independent and growth-process-dependent, [2]. Misfit dislocation is an example of growth-process-independent defects. The intrinsic stress $\sigma$ in the thin film is generated by two different mechanisms. The first one is the mismatch in lattice parameter between the thin film and the substrate. The next mechanism of the intrinsic stress is the mismatch of the thermal expansion coefficient between the film and the substrate. The intrinsic lateral residual stress may be very large, however, it is still considerably lower than the strength of the LPCVD (Low-Pressure-Chemical-Vapor-Deposition) poly-silicon films, which is widely used in microelectronics as well as micro-electro-mechanical systems (MEMS). Therefore, the thin film is still in the linear elastic range. If the original traction-free flat elastic film is subjected to a lateral bulk stress $\sigma$, as illustrated in Fig. 3a, then the first-order approximation of the stress for a wavy cosine surface, shown in Fig. $3 \mathrm{~b}$ is given by



Fig. 2. Perturbation of wavelength $\lambda$ and amplitude $q$ of a thin film of nominal thickness $h_{0}$ 
a

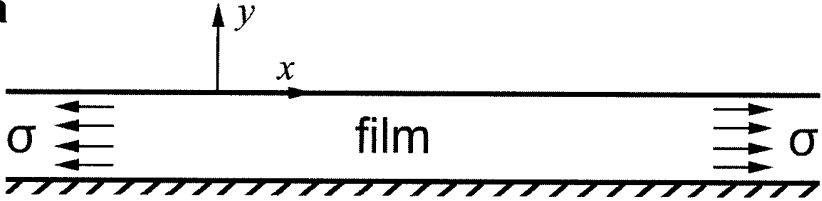

substrate
Fig. 3. a Traction-free flat surface under lateral stress, and b slightly undulating surface under lateral stress

$\sigma^{\prime}=\sigma\left(1-q \frac{4 \pi}{\lambda} \mathrm{e}^{\frac{2 \pi y}{\lambda}} \cos \frac{2 \pi x}{\lambda}\right)$

where $y$ is the vertical coordinate. In particular, the tangential component along the surface of the intrinsic stress is predicted as, [7],

$\sigma_{\tan }=\sigma\left(1-\frac{4 \pi q}{\lambda} \cos \frac{2 \pi x}{\lambda}\right)$.

Then the elastic strain energy density is given by

$w(x)=\frac{\sigma_{\tan }^{2}}{2 E}=\frac{\sigma^{2}}{2 E}\left(1-\frac{8 \pi q}{\lambda} \cos \frac{2 \pi x}{\lambda}\right)+O\left(q^{2}\right)$.

The strain energy with the variation of the amplitude of the undulation is

$\frac{\partial\left(\Delta W_{e}\right)}{\partial q}=\int_{0}^{\lambda} w(x) \cos \frac{2 \pi x}{\lambda} \mathrm{d} x=-2 \pi \frac{\sigma^{2}}{E} q+O\left(q^{2}\right)$,

so that the elastic energy variation per period is

$\Delta W_{e}=\int_{0}^{q} \frac{\partial\left(\Delta W_{e}\right)}{\partial q} \mathrm{~d} q=-\pi \frac{\sigma^{2}}{E} q^{2}$,

which does not depend on the wavelength, implying that stress tends to amplify surface perturbation of all wavelengths.

From (4) and (9) it is known that elastic strain energy decreases but surface energy increases when the flat film surface is perturbed into a wavy shape. If the stability of the epitaxial film on a substrate is determined primarily by the competition between surface energy and elastic strain energy, then by making minimum of the total free energy variation yields, [1],

$\lambda_{\mathrm{cr}}=\frac{\pi E \gamma_{0}}{\sigma^{2}}$,

which gives the critical value of the wavelength. The flat surface of the epitaxial film is stable if the actual wavelength of the perturbation is less than such critical value, but unstable if the characteristic length is greater than this value.

\section{3}

Role of van der Waals force in stability of film surface

If the van der Waals interactions between the film and the substrate are considered, then the variation of the volumetric free energy is 
$\Delta W_{v \mathrm{~d} W}=\left.\int_{0}^{\lambda} \frac{\mathrm{d} w_{v \mathrm{~d} W}}{\mathrm{~d} h}\right|_{h_{0}}\left(h-h_{0}\right) \mathrm{d} x+\left.\int_{0}^{\lambda} \frac{\mathrm{d}^{2} w_{v \mathrm{~d} W}}{\mathrm{~d} h^{2}}\right|_{h_{0}} \frac{\left(h-h_{0}\right)^{2}}{2} \mathrm{~d} x+\cdots$

The first term on the right-hand side of (11) is zero due to the mass conservation. Substituting (2) into (11) and neglecting the terms higher than the second-order, the dispersion energy in (11) becomes

$\Delta W_{v \mathrm{~d} W}=\left.\frac{\mathrm{d}^{2} w_{v \mathrm{~d} W}}{\mathrm{~d} h^{2}}\right|_{h_{0}} \frac{q^{2} \lambda}{4}$.

It is known, from Appendix, that the dispersion energy density of van der Waals interactions between two flat surfaces in the non-retarded regime $(D<20 \mathrm{~nm})$ is given by

$W_{v \mathrm{~d} W}(h)=-\frac{A}{12 \pi h^{2}}$,

where $A$ is the Hamaker constant. By substituting (13) into (12), we obtain

$\Delta W_{v \mathrm{~d} W}=-\frac{A q^{2} \lambda}{8 \pi h^{4}}$

The total free energy change in the thin film is

$\Delta W=\Delta W_{s}+\Delta W_{e}+\Delta W_{\nu \mathrm{d} W}=\frac{\pi^{2} \gamma_{0} q^{2}}{\lambda}-\frac{\pi \sigma^{2} q^{2}}{E}-\frac{A q^{2} \lambda}{8 \pi h^{4}}$

The stability of the thin film requires the minimum of the total free energy change, so that a quadratic equation for $\lambda$ is obtained from (15)

$\lambda^{2}+\frac{8 \pi^{2} h^{4} \sigma^{2}}{A E} \lambda-\frac{8 \pi^{3} \gamma_{0} h^{4}}{A}=0$

Therefore, the stable thickness of the thin-film is determined from the physical requirement of a single root of $\lambda$ in Eq. (16)

$h_{\text {sta }}=\frac{1}{\sigma}\left(-\frac{A \gamma_{0} E^{2}}{2 \pi}\right)^{1 / 4}$

which is the same as that obtained by another method in [6]. Equation (17) shows that the critical thickness of the film is inversely proportional to the applied stress, and only repulsive interaction $(A<0$, as shown in Fig. 4$)$ can stabilize the film. The film would be stable when

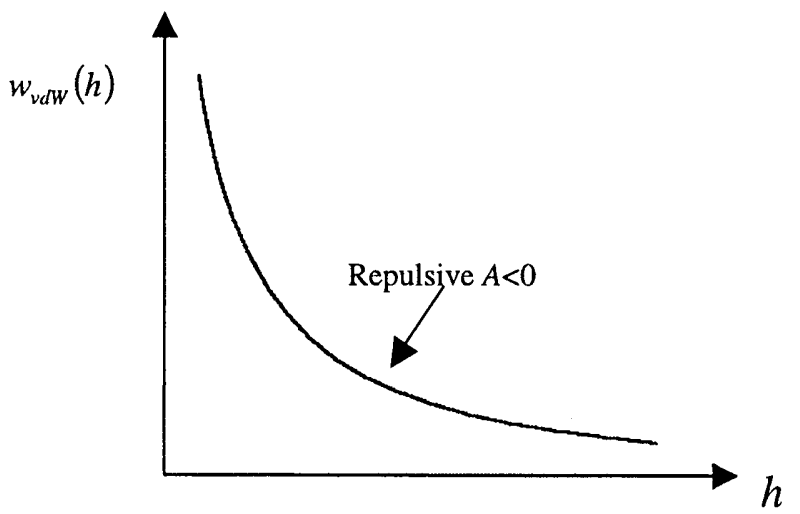

Fig. 4. Illustration of repulsive dispersion force 
$h \leq h_{\text {sta }}$.

The existence of solution of Eq. (16) requires

$\frac{64 \pi^{4} h^{8} \sigma^{4}}{A^{2} E^{2}}\left(1+\frac{A \gamma_{0} E^{2}}{2 \pi h^{4} \sigma^{4}}\right) \geq 0$

therefore we have the lower bound of the Hamaker constant as follows:

$A \geq-\frac{2 \pi h^{4} \sigma^{4}}{\gamma_{0} E^{2}}$

Thus the restriction on the Hamaker constant required by the stability of the thin film is

$-\frac{2 \pi h^{4} \sigma^{4}}{\gamma_{0} E^{2}} \leq A<0$

Under the restrictions of (18) and (21), the critical value of the wavelength of the undulation is given by the root of Eq. (16) which has the smaller value

$\lambda_{\mathrm{cr}}=\frac{4 \pi^{2} h^{4} \sigma^{2}}{|A| E}\left(1-\sqrt{1+\frac{A \gamma_{0} E^{2}}{2 \pi h^{4} \sigma^{4}}}\right)$.

In particular, when the thickness of the film happens to be the value in (17), then (22) reduces to the following simple form

$\lambda_{\mathrm{cr}}=\frac{2 \pi E \gamma_{0}}{\sigma^{2}}$.

By comparing (23) with (10), it is seen that at limit thickness of thin film the consideration of the van der Waals forces doubles the critical wavelength of the undulation of the thin film surface.

4

\section{Discussion and concluding remarks}

Equations (10) and (22) mean that the critical wavelength of the undulation does not depend on the amplitude $q$. The stable thickness of the film can be determined by consideration of van der Waals force, and the critical wavelength depends on the film thickness. On the other hand, the critical wavelength determined by the classic energetic conservation method does not depend upon the film thickness, and any film thickness seems stable.

If $|A| \gamma_{0} E^{2} /\left(2 \pi h^{4} \sigma^{4}\right)$ is small enough, from (22) it is seen that

$\lambda_{\mathrm{cr}} \sim \frac{4 \pi^{2} h^{4} \sigma^{2}}{|A| E}\left[1-\left(1+\frac{1}{2} \frac{A \gamma_{0} E^{2}}{2 \pi h^{4} \sigma^{4}}\right)\right]=\frac{\pi E \gamma_{0}}{\sigma^{2}}$,

which reduces to the case in which the role of van der Waals forces is not considered, by comparing with (10).

If the wavelength of perturbation $\lambda$ is taken as the characteristic length-scale, then the surface diffusion rate at which the shape evolves is determined by the temperature, diffusivity and this length scale. Actually, the normal velocity of the surface evolution is

$V_{n}=\frac{D_{s} \Omega^{2} S_{0}}{\kappa T} \nabla_{s}^{2}\left(\frac{1}{2} \sigma_{i j} \varepsilon_{i j}-\gamma_{0} K\right)$

where $D_{s}$ is diffusivity, $\kappa$ is the Boltzmann's constant, $T$ is absolute temperature, $K$ is the mean curvature, $\sigma_{i j}$ is the stress tensor component, $\varepsilon_{i j}$ is the strain tensor component, $\Omega$ and $S_{o}$ are atomic volume and number of atoms per unit area on the solid surface, respectively. We introduce nondimensional variables as follows: 
$x_{i}^{*}=\frac{x_{i}}{\lambda}, \quad u_{i}^{*}=\frac{u_{i}}{\left(\frac{\sigma}{E} \lambda\right)}, \quad \sigma_{i j}^{*}=\frac{\sigma_{i j}}{\sigma}, \quad \varepsilon_{i j}^{*}=\frac{\varepsilon_{i j}}{\left(\frac{\sigma}{E}\right)}, \quad t^{*}=\frac{t}{\left(\frac{B}{\lambda^{4}}\right)}$,

where $\sigma$ is the characteristic loading (for example the lateral residual stress). With these definitions, we have

$\nabla_{s}^{* 2}=\lambda^{2} \nabla_{s}^{2}, \quad K^{*}=\lambda K$,

and the nondimensional normal velocity of the surface evolution is

$V_{n}^{*}=\nabla_{s}^{* 2}\left(\Lambda \frac{\sigma_{i j}^{*} \varepsilon_{i j}^{*}}{2}-K^{*}\right)$

where

$\Lambda=\frac{\lambda \sigma^{2}}{E \gamma_{0}}$

is the sole dominating nondimensional parameter. It is the ratio of strain energy density to surface energy, therefore it is termed "bulk-surface number". The dependence of the mass diffusion on the wavelength of perturbation is reflected by such number. Furthermore, the nondimensional time-scale is given by

$t^{*}=\frac{\lambda^{4}}{\left(\frac{D_{s} \Omega^{2} S_{0} \gamma_{0}}{\kappa T}\right)} t$

The characteristic time for surface diffusion depends on this natural length-scale according to $\lambda^{4}$. An implication of this observation is that morphology evolution is not expected to occur on macroscopic scale, [4].

It is noted that Eq. (10) can be expressed as $\Lambda_{\mathrm{cr}}=\pi$, and Eq. (23) can be expressed as $\Lambda_{\mathrm{cr}}=2 \pi$.

The theoretical analysis in the present paper can only be used to interpret the stability of undulated thin films as shown in Fig. 1a. Further analysis is needed for the stability of thin films with both undulation and strain contrast as shown in Fig. 1 b.

It is noted that all the existing theoretical analyses for morphological stability of epitaxial thin elastic film have used the assumption that the substrate is flat. This might be true for thick films. Nevertheless, if one considers an elastic film of tens or even hundreds of nanometers thick, the roughness of the substrate would play an important role in the epitaxial film deposition. Further experimental and theoretical studies will be interesting and of practical importance.

It is also noted that a one-dimensional problem has been discussed in the present paper. For a two-dimensional problem, on the other hand, Poisson's ratio $v$ needs to be included in the lateral residual stress. Suppose that a film of some crystal class and lattice parameter $a_{f}$ grows coherently with a nominally uniform thickness on a surface of a second (relatively thick) substrate material of the same crystal class and orientation, but with lattice parameter $a_{s}$. The lattice mismatch (misfit) parameter in a dislocation-free film is $\varepsilon_{m}=\left(a_{s}-a_{f}\right) / a_{f}$, this is a fundamental system parameter and is numerically equal to the elastic strain in the film. Correspondingly, a biaxial stress $\sigma_{m}=E \varepsilon_{m} /(1-v)$ is generated in the film. For this problem, the film surface can be perturbated into

$h=h_{0}+q \cos \frac{2 \pi x}{\lambda} \cos \frac{2 \pi z}{\lambda}$.

To sum up, the present paper mainly studies the influence of van der Waals force on the stability of epitaxial thin films on a substrate. The dispersion interactions can compete with the stress in thin films. It is shown that only van der Waals force with negative Hamaker constant $(A<0)$ tends to stabilize the film, while van der Waals force with positive Hamaker constant 
$(A>0)$ tends to destabilize. The lower bound of the Hamaker constant is also obtained for the stability of thin films. The critical value of the undulation wavelength is found to be a function of both film thickness and external stress. The characteristic time-scale for surface mass diffusion scales to the fourth power to the wavelength of the perturbation.

\section{Appendix}

Van der Waals interactions for the most common geometries

Geometries Interaction law (per unit area)

\begin{tabular}{ll}
\hline Two spheres & $-\frac{A}{6 D} \frac{R_{1} R_{2}}{R_{1}+R_{2}}$ \\
Sphere-flat surface & $-\frac{A R}{6 D}$ \\
Two flat surfaces & $-\frac{A}{12 \pi D^{2}}$
\end{tabular}

$R$ is the radius of sphere, $D$ the distance between interacting bodies, and $A$ the Hamaker constant.

\section{References}

1. Gao, H.; Nix, W.D.: Surface roughening of heteroepitaxial thin films. Annu Rev Mat Sci 29 (1999) 173-209

2. Mahajan, S.: Defects in semiconductors and their defects on devices. Acta Mat 48 (2000) 137-149

3. Cullis, A.G.; Robbins, D.J.; Pidduck, A.J.; Smith, P.W.: The characteristics of strain-modulated surface undulations formed upon epitaxial $\mathrm{Si}_{1-\mathrm{x}} \mathrm{Ge}_{\mathrm{x}}$ alloy layers on Si. J Cryst Growth 123 (1992) 333-343

4. Freund, L.B.: The mechanism of electronic materials. Int J Solids Struct 37 (2000) 186-196

5. Israelachvili, J.N.: Intermolecular and Surface Forces. 2nd edn. Academic Press, 1991

6. Suo, Z.; Zhang, Z.: Epitaxial films stablized by long-range forces. Phys Rev B58 (1998) 5116-5120

7. Gao, H.: A boundary perturbation analysis for elastic inclusions and interfaces. Int J Solids Struct 28 (1991) 703-725 